\title{
Propuesta de modelo de gestión del conocimiento y su impacto en la eficiencia operacional. Caso Universidad Tecnológica de Tula Tepeji, área Mecatrónica
}

\section{Proposed knowledge management model and its impact on operational efficiency. Case of the Technological University of Tula Tepeji, Mechatronics area}

\author{
MARTÍNEZ-SÁNCHEZ, Sergio*† \& LARA-GÓMEZ, Graciela \\ Cuerpo Académico Optimización de Procesos Productivos de la Universidad Tecnológica Tula-Tepeji /Estudiante de \\ Doctorado del Instituto Tecnológico Latino Americano \\ Cuerpo Académico Organización y Desarrollo de la Universidad Autónoma de Querétaro
}

ID $1^{\text {er }}$ Autor: Sergio, Martínez-Sánchez / ORC ID: 0000-0003-0838-3184, Researcher ID Thomson, Y-3342-2018, CVU CONACYT ID: 510245

ID $1^{\mathrm{er}}$ Coautor: Graciela, Lara-Gómez / ORC ID: 0000-0001-9984-7372, CVU CONACYT ID: 99837

DOI: $10.35429 / J E S C .2019 .8 .3 .16 .22$

Recibido: 10 de Marzo, 2019; Aceptado 30 de Junio, 2019

\section{Resumen}

El objetivo principal de la investigación es proponer un modelo de gestión del conocimiento para el programa educativo de mecatrónica de la Universidad, que permita incrementar la eficiencia y eficacia de sus operaciones, y/o, para que puedan ser realmente procesos que permitan incrementar el conocimiento efectivo y práctico de todo el personal de la institución, propiciando la productividad y competitividad de los egresados y cuerpos académicos, siendo estos detonantes del desarrollo social y económico de la región, asimismo incrementando la capacidad de respuesta ante un mundo globalizado y dinámico. La presente investigación se enmarca dentro del tipo de investigación descriptiva, bajo la modalidad de un proyecto factible, ya que se ofrece una solución viable a un problema de tipo práctico; además, se apoya en el diseño no experimental transeccional descriptivo.

Gestión del conocimiento, Acreditación, Programa educativo

\begin{abstract}
The main objective of the research is to propose a knowledge management model for the educational mechatronics program of the University, which allows to increase the efficiency and effectiveness of its operations, and / or, so that they can be really processes that allow to increase knowledge effective and practical for all staff of the institution, promoting the productivity and competitiveness of graduates and academic bodies, to be triggers of social and economic development in the region, also increasing the ability to respond to a globalized and dynamic world. The present investigation is framed within the type of descriptive investigation, under the modality of a feasible project, since a viable solution to a problem of practical type is offered; In addition, it is based on the non-experimental descriptive transectional design.
\end{abstract}

Knowledge management, Accreditation, Educational program

Citación: MARTÍNEZ-SÁNCHEZ, Sergio \& LARA-GÓMEZ, Graciela. Propuesta de modelo de gestión del conocimiento y su impacto en la eficiencia operacional. Caso Universidad Tecnológica de Tula Tepeji, área Mecatrónica. Revista de Ciencias de la Educación. 2019. 3-8: 16-22

\footnotetext{
* Correspondencia del Autor (correo electrónico: sergio.martinez@uttt.edu.mx)

$\dagger$ Investigador contribuyendo como primer autor.
} 


\section{Introducción}

Ante un mundo globalizado, que está cambiando vertiginosamente de una manera tan compleja que es difícil discernir cuales son los cambios más significativos y los que tendrán mayor influencia en la sociedad actual y del mañana. Estos cambios y el desarrollo de nuevas tecnologías, en la última década, se ha dado impulso notable, las comunicaciones, la optimización de los recursos, al impacto ambiental, las energías renovables, los cuales hasta hace pocos años, no se habían tomado en cuenta. Ante estos retos surge en 1991, un modelo de universidad, las tecnológicas (López, 2008).

Estas instituciones tienen como finalidad dar respuesta a las demandas del sector industrial, ya que nacen vinculadas con el sector productivo. En este contexto desde su nacimiento han operado de esta forma, aun cuando se tenía cierta desconfianza de los programas educativos y por ende de los egresados, en relación a la capacidad de dar respuesta y ante los retos que el propio sector laboral y los cambios tecnológicos exigían. En la última década del siglo pasado eran considerados como estudiantes de nivel medio superior, pero con el tiempo se fueron posicionando y obteniendo el reconocimiento oficial de cada uno de los diversos sectores de la sociedad.

Ante la actual dinámica, los cambios tecnológicos y la globalización, hacen que las oportunidades de inserción laboral de los egresados de estas instituciones sean cada día más competitiva, y aunado a la carencia de capacidades que presentan al egresar de las universidades tecnológicas, como lo declara (Silva, 2006). "Los empresarios dieron la más baja valoración a la capacidad analítica y lógica de los jóvenes, a su habilidad para solucionar problemas, para trabajar de manera autónoma y ejercer un liderazgo en el trabajo". (Villa, 2018).

Por otra parte, son considerados con actitudes muy positivas relacionadas con la responsabilidad, la disciplina, el respeto y la disposición a aprender. Pero son poco propositivos, faltos de iniciativa y carentes de compromiso con la empresa. Es una característica que presentan los estudiantes de corte ingenieril en el programa educativo de la institución donde se realizará la investigación.
La variabilidad del entorno social, económico, político, el conocimiento y las necesidades más apremiantes, propician que el sector educativo necesite una profunda transformación que permita atender las demandas de la sociedad (Márquez, 2017).

Ante este panorama descrito y con la misión de fomentar la eficiencia y eficacia de los procesos propios de la institución, donde se propicie la mejora continua, que apoyan a la productividad del programa educativo, es considerado fomentar la evaluación-acreditación de los procesos, ante organismos acreditadores. Por lo que programa Nacional de Educación 2001-2006 (PRONAE) reconoció que la evaluación externa y la acreditación de la educación superior son medios fundamentales para fomentar la mejora y el aseguramiento de su calidad, y promover la equidad al permitir detectar desigualdades de calidad en los programas educativos, así como en los niveles de aprendizaje alcanzados por los estudiantes. (Rubio, 2007). Con lo anterior descrito en el 2006 las universidades tecnológicas, inician el proceso de acreditación ante organismo acreditadores reconocidos por COPAES, con la finalidad de asegurar la calidad de los programas educativos evaluados, fortaleciendo los procesos educativos y administrativos y de investigación.

La acreditación nos permite garantizar que se cumplen con los estándares de calidad y es primordial para mejorar el proceso educativo, en este sentido se considera que con la implementación, mejorará la comunicación y solucionará los problemas relacionados a la falta de información que los individuos pueden tener en torno a la calidad de los servicios educativos, de tal forma que su principal función de la acreditación es otorgar reconocimiento público a la institución y a los programas educativos que cuentan con un mínimo estándar de calidad, lo que permite en su momento el acceso a la información de todos los involucrados en los diferentes procesos que conforman la institución.

Sin embargo, los resultados del impacto de la acreditación a su llegada a la educación y sus problemas que presentan como lo describen. (Márquez, 2017), han tenido avances significativos (Buendía, 2014). 
Los resultados obtenidos en su investigación, muestran que los procesos de acreditación no han generado efectos decisivos en la mejora de la calidad de los programas educativos debido a la influencia de otros factores, si bien es cierto, se ha avanzado en una cultura de la evaluación (De la Garza, 2013).

Esta evaluación-acreditación del programa educativo, no ha contribuido significativamente para que los procesos sean eficientes y eficaces, que propicien un Programa Educativo, competitivo, por ende un egresado capaz de dar respuesta a los cambios tecnológicos que la sociedad demanda.

En este contexto la gestión del conocimiento no es un tema actual, desde hace décadas se trabaja, pero con un enfoque hacia el sector industrial, no obstante en tiempos actuales, la gestión del conocimiento se viene aplicando a la instituciones de educación, aun sabiendo que las instituciones son generadoras mismas del conocimiento.

Ahora bien, las instituciones universitarias que tienen como tarea la creación de conocimiento para el desarrollo sustentable deberían ser un ejemplo de creación de valor a su interior (López et al., 2015). Por esta razón, es necesario desarrollar un modelo de gestión del conocimiento al interior del programa educativo acreditado de mecatrónica que permitan a todas las personas de la institución desde el nivel jerárquico más bajo hasta el nivel más alto, disponer de todo el potencial del conocimiento que se encuentra disperso en la institución, agruparlo, tenerlo y dofundirlo.

Esto implica crear y desplegar una estrategia de gestión del conocimiento que integre cinco acciones fundamentales: entender necesidades y oportunidades del conocimiento; construir conocimientos relevantes para el crecimiento; organizar y distribuir el conocimiento de la organización; crear condiciones para la aplicación del conocimiento de la institución y explotar el conocimiento. (Nonaka, \& Ichijo. p.1 2001)

\section{Problema}

La competitividad no solo es aplicable al sector empresarial ahora está en los entornos educativos, siendo el proceso a través del cual se orienta y conduce la labor docenteinvestigación-administrativa de la institución y sus relaciones en cuanto al entorno del aprendizaje, con miras a conseguir los objetivos institucionales, mediante el trabajo coordinado de todos los miembros del programa educativo, a fin de ofrecer un servicio de calidad, y coordinar las distintas tareas y funciones de los miembros hacia la consecución de sus objetivos.

Tomando en cuenta el conjunto de habilidades directivas; orientadas a planificar, organizar, coordinar y evaluar la gestión estratégica de aquellas actividades necesarias para alcanzar eficacia y la eficiencia de los procesos de aprendizaje, investigación, administración, y la trascendencia cultural, generando un pensamiento sistémico, haciendo grandes cambios de paradigmas y costumbres, ya no se basa en la concepción de enseñanza aprendizaje como transmisión y observación sino que en la actualidad, está orientada a un modelo activo y participativo, permitiendo establecer nuevas estrategias para el aprendizaje: un "aprendizaje significativo" y transformador.

Una condición necesaria para lograr estos aprendizajes es que la metodología que se utilice propicie una interacción sistémica, de toda la estructura de la institución, cultural, liderazgo, así como los procedimientos de aprendizaje, las actitudes de las personas, la capacidad de trabajo en equipo o en conjunto, de todos los elementos que componen el proceso, identificando aquellos factores de influencias, que en cierto modo no forman parte del proceso pero influyen en un resultado final. Por ejemplo considerando las actividades de competitividad, las cuales permiten a los estudiantes desarrollar su potencial heurístico; su capacidad de análisis, de síntesis; convirtiéndose en verdaderos innovadores y competitivos.

En el contexto de la educación existe una concepción esencialmente lineal de la actividad competitiva, cuyas prácticas de aprendizaje y de investigación, tienden a utilizar distintas metodologías de enseñanza-aprendizaje. 
En algunos casos como estrategias para solucionar problemas propios del conocimiento, en otras con un resultado poco significativo que presentan un desconocimiento total del perfil, de los objetivos institucionales de su profesión y del entorno donde desarrollaran su actividad profesional. Esto hace que presenten una desventaja competitiva ante los diversos sectores empresariales.

Frente a ello, la tendencia actual en el ámbito de la educación superior, es de mayor énfasis, en las actividades en caminadas a la generación del conocimiento, en este sentido aun cuando la universidad cuya función es la transferencia misma del conocimiento, en este sentido las instituciones dentro de su responsabilidad social está la de crear una conciencia entre la comunidad universitaria que le generación misma del conocimiento implica una forma de ver la realidad, de asumir responsabilidades, compartir el conocimiento; siendo esta una tendencia de forma de vida, frente a la cotidianidad; o sea, viene a ser una manera de relacionarse en la sociedad y el entorno donde se ubica la institución.

En ese contexto, se percibe que el sistema educativo en México también ha sufrido grandes transformaciones en la primera década del siglo XXI. Actualmente el debate en el seno propio de los órganos acreditadores sobre la importancia de evaluar y acreditar los procesos educativos, resaltando la eficiencia y eficacia misma de los procesos y del impacto que trae consigo así como el reconocimiento ante los diversos sectores de la sociedad.

Además, los estudiantes, por su naturaleza predominantemente activa, necesitan el aprendizaje integral para construir su propia identidad y subjetividad, otorgándoles la ventaja que les permita un mejor desenvolvimiento en todas y cada una de sus actividades profesionales. Desarrollar en ellos mismos la seguridad y la habilidad de entender el conocimiento mismo y su responsabilidad de usarlo en beneficio de su entorno social y laboral. Analizar y sustentar cada una de las decisiones que tome en su momento, así como el conocimiento y la comprensión progresiva de la realidad. En este mismo orden de ideas, se considera que la generación del conocimiento compartido al servicio de todos, propicia el interés por conocer más, permite desarrollar la seguridad en cada uno de los individuos que conforman la institución.
La gestión del conocimiento mismo es una forma de ver la realidad es de participación o de actividad asociativa, donde no hay una verdadera división de roles u organización en las relaciones sociales en cuestión; cada integrante conoce, comparte, se vuelve competitivo en su entorno.

Asimismo, se puede percibir en instituciones educativas de nivel superior que cuenten con programas educativos acreditados ante consejos acreditadores en el estado de Hidalgo, que cada vez tiene más relevancia e importancia la acreditación, ya sea para tener el reconocimiento de los diversos sectores de la sociedad y para eficientar los procesos de aprendizaje-investigación y administrativos, por comprenderse esenciales para que se den verdaderos espacios en donde el estudiante, se forme bajo un proceso eficiente y eficaz. Esta sería la explicación del aumento del número de programas educativos acreditados, permitiendo un cambio transcendental en la docencia y en la investigación

\section{Justificación}

Se propone desarrollar un modelo de gestión del conocimiento que logre alcanzar la articulación entre los diversos procesos acreditados del programa educativo de Mecatrónica de la Universidad Tecnológica de Tula-Tepeji, que permita generar el conocimiento y compartirlo. A tal fin parte la siguiente pregunta ¿Por qué el programa educativo de mecatrónica, se presentan problemas de baja productividad académica, si cuenta con la acreditación de un organismo de alto nivel, que acredita las competencias? Con el propósito de responder a esta pregunta revisamos, en primer lugar, se debe conceptualizar el conocimiento de los integrantes que forman parte de los diversos procesos, desde una perspectiva histórica, particularmente partiendo de un diagnóstico, desde el punto de vista del aprendizaje.

Para las instituciones educativas de hoy, ser competitivas requiere de aceptar los cambios y asumir los retos de este mundo cambiante, en el desarrollo de nuevas tecnologías con una dinámica vertiginosa y un mundo que tiende a la globalización mediante las tecnologías de la información. 
Un desafío es tener conciencia de que las instituciones ya no son vistas como máquinas, sino como organismos vivientes con necesidades físicas, emocionales, mentales y espirituales que deben ser consideradas, para tener capacidad de respuesta.

Por esta razón, es necesario desarrollar mecanismos que permitan a todas las personas de la institución desde el nivel jerárquico más bajo hasta el nivel más alto, disponer de todo el potencial del conocimiento que se encuentra disperso en la institución, agruparlo y tenerlo. Esto implica crear y desplegar una estrategia de gestión del conocimiento que integre cinco acciones fundamentales: entender necesidades y oportunidades del conocimiento; construir conocimientos relevantes para el crecimiento; organizar y distribuir el conocimiento de la organización; crear condiciones para la aplicación del conocimiento de la institución y explotar el conocimiento.

Para asegurar que la gestión del conocimiento obtenga la eficiencia y eficacia de los procesos del programa educativo de la institución, es necesario desarrollar la capacidad de realizar procesos de transferencia de conocimientos, de manera efectiva, alienados con las normas de calidad y de acreditación con que actualmente el programa educativo cuenta. En este sentido, elementos como: la adquisición y apropiación de conocimientos, la solución de problemas, la implementación e integración de soluciones, la experimentación y generación de prototipos, son esenciales para el logro de los objetivos.

Podemos concluir que a través de los procesos diarios realizados dentro de una institución educativa se genera una gran cantidad de conocimiento, este conocimiento en muchos casos puede ser utilizado para la misma organización en algún otro proceso relacionado. En la mayoría de las ocasiones, este conocimiento no se documenta ni se almacena en algún lugar de la organización, quedando solamente en la memoria de las personas que lo experimentaron, para después quedar normalmente en el olvido si este no se utiliza de manera periódica
El objetivo de la implementación de un sistema de gestión del conocimiento, es contar con un sistema metodológico que apoye a la creación, almacenamiento, compartición y utilización de ese conocimiento generado dentro de la organización, de una forma benéfica, a través de la utilización del mismo por todos los integrantes de la institución, para eficientar los procesos educativos.

\section{Hipótesis}

Un modelo de gestión del conocimiento podrá mejorar la eficiencia operacional de la Universidad Tecnológica de Tula-Tepeji, en el área de mecatrónica.

\section{Objetivos}

\section{Objetivos específicos}

- Formular una estrategia de alcance institucional, para el desarrollo, adquisición y aplicación del conocimiento

Promover la mejora continua de los procesos del programa educativo, enfatizando la generación y utilización del conocimiento, para hacer eficientes $y$ eficaces los procesos

- Desarrollar el modelo de gestión del conocimiento acorde al programa educativo de mecatrónica de la Universidad Tecnológica de Tula Tepeji.

\section{Materiales y métodos}

Para este trabajo se realizó una investigación bibliográfica del tipo de investigación descriptiva, bajo la modalidad de un proyecto factible, ya que se ofrece una solución viable a un problema de tipo práctico; además, se apoya en el diseño no experimental transeccional descriptivo.

Los temas, en el cual diversos investigadores de las organizaciones coinciden en las dinámicas de los cambios en la tecnología. Así mismo, ante el constante desarrollo y avance tecnológico en un mundo globalizado en las últimas décadas, las organizaciones han tenido que verse obligadas a adoptar nuevas herramientas tecnológicas como la gestión del conocimiento y la capacitación de su personal en el uso eficiente y eficaz de dichas herramientas. 
Como lo describe en su investigación (Contreras, 2018), en su investigación coincide con investigadores que la gestión del conocimiento como un elemento promotor de la productividad y competitividad, es ahí donde toma relevancia un tema ampliamente estudiado, pero que debe ser de principal interés

Por gestión del conocimiento se entiende como el procesos continuo de aprendizaje en una organización para hacer más creativos e innovadores a los miembros de la institución a efectos de que éstos sean más precisos en la toma de decisiones, puedan brindar una respuesta más rápida a las necesidades del sector laboral y de investigación, y obtengan un desarrollo sostenible que los haga más competitivos. (Taype, 2015)

\section{Conclusiones}

El programa educativo acreditado de la institución, representa una realidad distinta de los que establecen los organismos acreditados. A pesar de la importancia que tiene dicha acreditación, se percibe una escasa aplicación de las mismas, producto del escaso conocimiento que se tiene sobre los criterios del proceso de acreditación, en relación al marco de referencia, y la manera de conducirlas. Se considera, por ejemplo, en forma equivocada, que estas actividades por si solas mejorarán el aprendizaje de cada uno de los integrantes que ejecuta, actúa y aplica de forma distinta a las políticas y normas establecidas, derivado del desconocimiento mismo.

El conocimiento empírico que traen consigo mismo el personal, efectivamente lo comparten con un grupo reducido de personas, sin embargo no es compartido con el resto del personal, estudiantes, y administrativo. Así que cuando emigran se llevan consigo mismo el valioso conocimiento, al no contar con un proceso de almacenamiento y difusión.

Esto explicaría las razones, de la baja eficiencia y eficacia de los procesos de aprendizaje-investigación y administración, que impactan en la producción académica del programa educativo, además del por qué la desproporcional diferencia de conocimientos entre la comunidad educativa, asimismo la desventaja que se tiene con respecto a la formación académica de instituciones educativas del sector privado.
Ante esta situación, en el comité interno del programa educativo de mecatrónica, preocupado por la baja productividad, no obstante el contar con una acreditación en el marco de referencia respetivo, deberían propiciar mejores resultados.

Por lo antes expuesto, en este contexto, es necesario analizar en qué medida la participación de todos y cada uno de los integrantes que participa en el proceso, con el propósito de plantear una solución donde los actores o protagonistas no sean únicamente los estudiantes y el docente, sino también, todo el personal administrativo y de apoyo. De esta forma, se pretende integrar a través de un modelo de gestión del conocimiento que permita interconectar a través de los canales establecidos de comunicación, todos los elementos que conforman el proceso institucional, así como los diversos en los diversos sectores de la sociedad.

En concordancia con los nuevos enfoques, tendencias y objetivos de las políticas del ISO 9000, de la Institución Educativa, del marco de referencia del organismo acreditador y del reglamento propio de la institución el cual tiene el reto de mejorar la calidad y competitividad de todos los integrantes de la comunidad educativa.

\section{Referencias}

Castillo-Zúñiga, J., Medina-León, A., MedinaNogueira, D., Medina-Nogueira, Y. E., \& Assafiri-Ojeda, Y. E. (2019). Modelo de gestión del conocimiento para el cultivo de Cacao en Vinces. Ingeniería Industrial, 40(1), 48-58.

Erik Márquez \& Zulma Raquel. (Diciembre 2016). El Impacto de la Acreditación en la Mejora de la Calidad de los Programas Educativos que Ofrece la Universidad Autónoma de Tamaulipas: Un Estudio de Caso. Revista Iberoamericana de Evaluación Educativa, 10, p. 65-83.

F. Barroso, (2011), Gestión del conocimiento en instituciones de educación superior y centros de investigación científica en el estado de Yucatán, México, Anfeca.

I. Inche \& A. Chung, (2004). Indicadores de gestión del conocimiento en la facultad de ingeniería industrial. Facultad de Ingeniería Industrial, Volumen 7, p. 41-45. 
J. Acosta, (2011), Implementación de un modelo de gestión del conocimiento en los procesos organizacionales: Caso Disan S.A. Pontificia Universidad Javeriana, Bogota Colombia.

Janet López Barrios. (Junio 2008). Las Universidades Tecnológicas Mexicanas un modelo eficaz, una inversión pública exitosa, un sistema a fortalecer. Tiempo de educar, 9, p. 147-152.

K. Barrios \& J. Acosta. (2018). Gestión del conocimiento y capacidad de innovación. Bogota Colombia: Mejoras.

L. Cortés. (2016). La gestión del conocimiento y la sostenibilidad de la acreditación del Hospital Pablo VI Bosa de Bogotá. SIGNOS, $8 \mathrm{~N}^{\circ} 1$, p.87-103.

L. Villa. (Marzo 2008). La calidad educativa de las universidades tecnológicas. Su relevancia, su proceso de formación y sus resultados. Revista de la Educación Superior, 37, 143-152.

Landazábal, M. S. C., Ruiz, C. G. A., Álvarez, Y. Y. M., \& Padilla, H. E. C. (2019). Lean manufacturing: 5 sy TPM, herramientas de mejora de la calidad. Caso empresa metalmecánica en Cartagena, Colombia. Signos: Investigación en sistemas de gestión, 11(1), 7186.

Munevar, A., Paola, D., Luna, G., \& Elena, L. (2019). Modelo de procesos para la gestión de peticiones, quejas, reclamos y sugerenciasPQRS de la Superintendencia de Sociedades bajo metodología business process managementBPM.

N. Nofal. (2007). La gestión del conocimiento como fuente de innovación. Escuela de Administración de Negocios, Volumen 61, p. 77-87.

Nonaka, \& Ichijo. (2001). Facilitar la creación del conocimiento. México D.F.: OXFORD.

Ortiz, M. M, (2019). Propuesta de un Modelo de Gestión de Infraestructura Hospitalaria mediante Facility Management para Colombia.

Probabilidad y estadística para ingenieros, Editorial Prentice - Hall 9. Erwin Kreyszing, Estadística matemática, Editorial Limusa 10. Spiegel Murray R, Probabilidad y estadística, Editorial Mc Graw - Hill
Probabilidad y estadística para ingenieros. Editorial Interamericana 6. Murria Spiegel, John Schiller, R. Alu Srinivasan.

Probabilidad y estadística. Editorial Mc Graw Hill 7. Meyer L. P, Probabilidad y aplicaciones estadísticas, Editorial Fondo Educativo Interamericano 8. Irwin Miller, John E. Freuno

Rodriguez Africano, J., Mantilla Toledo, J. A., Castro, P., Ashner, S., Felipe, A., \& Bedoya, V. (2019). Propuesta de diseño de un sistema de gestión de compras para la empresa Daniel Bejarano Arquitectos.

Taype Molina Martín. (2015, septiembre 28).5 tendencias en la gestión del conocimiento. 